\title{
Gut microbiota: effect of pubertal status
}

\author{
Xin Yuan, Ruimin Chen ${ }^{*} \mathbb{D}$, Ying Zhang, Xiangquan Lin and Xiaohong Yang
}

\begin{abstract}
Background: The make-up of gut microbiota at different puberty stages has not been reported. This cross-sectional study analyzed the bio-diversity of gut microbiota at different puberty stages.

Result: The subjects (aged 5-15 years) were divided into non-pubertal ( $n=42$, male\%: 66.7\%) or pubertal groups ( $n=47$, male\%:44.68); in both groups, Firmicutes, Bacteroidetes and Proteobacteria were the dominant phylum. There was no difference of alpha- and beta-diversity among disparate puberty stages. Non-pubertal subjects had members of the order Clostridiales, family Clostridiaceae, genus Coprobacillus which were significantly more prevalent than puberty subjects. Also, the pubertal subjects had members of class Betaproteobacteria, order Burkholderiales which were significantly more prevalent than the non-pubertal subjects. Their relative abundance was independent of BMI-Z. In the pubertal subjects, the abundance of genus Adlercreutzia, Ruminococcus, Dorea, Clostridium and Parabacteroides was associated with the level of testosterone.

Conclusions: This is the first report of the diversity of gut microbiota at different puberty stages. The various species of gut microbiota changed gradually associated with puberty stages. Differences in gut microflora at different pubertal status may be related to androgen levels.
\end{abstract}

Keywords: Puberty, Children, Adolescent, 16 s rRNA, Gut microbiota

\section{Background}

Puberty constitutes an important phase of life, which is associated with profound physiological changes related to sexual maturation during the transition toward adulthood. These somatic developmental changes are predominantly driven by hormones and are accompanied by psychological adjustment. Therefore, this dynamic period represents an unparalleled opportunity to assess potential hormonal impacts on gut microbiota [1]. Previous studies pointed out that human gut microbiota was adult-like after the first 3 years of life and were relatively stable $[2,3]$. However, although healthy pre-adolescent children (ages 7-12 years) and adults harbored similar numbers of taxa and functional genes, their relative composition differed significantly [4]. Nonetheless, Enck et al. conducted a large-scale study, using conventional

\footnotetext{
*Correspondence: chenrm321@163.com
Department of Endocrinology, Genetics and Metabolism, Fuzhou Children's

* Correspondence: chenrm321@163.com Hospital of Fujian Medical University, NO.145, 817 Middle Road, Fuzhou 350005, China
}

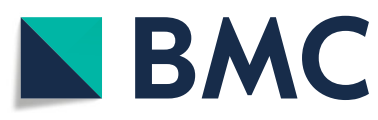

(c) The Author(s). 2020 Open Access This article is licensed under a Creative Commons Attribution 4.0 International License, which permits use, sharing, adaptation, distribution and reproduction in any medium or format, as long as you give appropriate credit to the original author(s) and the source, provide a link to the Creative Commons licence, and indicate if changes were made. The images or other third party material in this article are included in the article's Creative Commons licence, unless indicated otherwise in a credit line to the material. If material is not included in the article's Creative Commons licence and your intended use is not permitted by statutory regulation or exceeds the permitted use, you will need to obtain permission directly from the copyright holder. To view a copy of this licence, visit http://creativecommons.org/licenses/by/4.0/. The Creative Commons Public Domain Dedication waiver (http://creativecommons.org/publicdomain/zero/1.0/) applies to the data made available in this article, unless otherwise stated in a credit line to the data.

colony plating to assess numbers of several bacterial genera, found no noticeable changes in children between 2 and 18 years old [5]. Recently, with the expansion and availability of bacterial DNA sequencing technology, a study compared the distal intestinal microbiota composition between adolescents (11-18 years of age) and adults, founding the abundance of the genera Bifidobacterium and Clostridium was statistically significant higher in adolescent samples compared with adult samples [6].

Every organ is affected by the extensive changes in circulating hormone during puberty $[7,8]$. We postulated that these marked changes in hormone levels would alter the intestinal flora. No previous microbiota study has explored the full span of growth from pre-puberty to late puberty. Such information is instructive given the association between gut microbiota during growth and adult disease risk [9]. To this end, we utilized $16 \mathrm{~s}$ rRNA gene sequencing to compare fecal microbiota profiles from 
pre-puberty to adolescence, ranging in age from 5 to 15 years.

\section{Results}

\section{Study subjects}

The mean age of the 89 participates was $9.75 \pm 1.92$ years (ranging from 5.5 to 14.3 years) and $55.06 \%$ were boys. The majority $(73.03 \%)$ were obese based on BMI, and 26.97\% had normal BMI.

Based on puberty status, the subjects were divided into non-pubertal group $(n=42,66.7 \%$ male) and pubertal group ( $n=47,44.68 \%$ male). The average age was $8.36 \pm$ 1.64 years and $10.99 \pm 1.15$ years, respectively. There was no statistical difference in BMI-Z scores or dietary habits between the two groups ( $p=0.783$ and 0.641 , respectively). The non-pubertal group was further subdivided into a younger children group $(n=18,66.7 \%$ male $)$ and pre-pubertal group ( $n=24,66.7 \%$ male). And the pubertal group classified as early ( $n=18,77.8 \%$ male), middle ( $n=14,35.7 \%$ male), late $(n=15,13.3 \%$ male). Of the 40 girls, 21 had E2 measured, 2 were non-pubertal with a level of $\mathrm{E} 2<5 \mathrm{pg} / \mathrm{ml}$, and 19 were pubertal with a level of E2 $33.68 \pm 35.80 \mathrm{pg} / \mathrm{ml} ; 21$ of the 49 boys had T measured, 6 were non-pubertal with a level of $\mathrm{T} 5.10 \pm 4.16$ $\mathrm{ng} / \mathrm{dl}$, and 15 were pubertal with a level of $\mathrm{T} 83.20 \pm$ $98.55 \mathrm{ng} / \mathrm{dl}$. There was no statistical difference in BMI-Z scores, mode of birth, feeding patterns or dietary habits among the groups $(p>0.05)$. Tables 1,2 and Table S1, S2 describes the characteristics of the subjects.

\section{Core microbiota in all the subjects}

With 16S rRNA gene sequencing, 671 discrete bacterial taxa (OTUs) were identified. Most of these species belonged to the "shared" category of those common to multiple but not all samples. We also identified a "core" of 557 species shared among all fecal samples. The nonpuberty group had 49 unique species and the puberty group had 66 unique species (Fig. 1). The core microbiota was dominated by phylum Firmicutes, Bacteroidetes and Proteobacteria in both the non-pubertal and pubertal groups (Fig. 2 and Table S3).

Table 1 Clinical characteristics of the study population divided by puberty status

\begin{tabular}{llll}
\hline & Non-puberty $(\boldsymbol{n}=\mathbf{4 2})$ & Puberty $(\boldsymbol{n}=\mathbf{4 7}$ & $\boldsymbol{P}$ value \\
\hline Age (years) & $8.36 \pm 1.64$ & $10.99 \pm 1.15$ & $<0.001$ \\
Gender (male\%) & $66.7 \%$ & $44.68 \%$ & 0.054 \\
Height $(\mathrm{cm})$ & $132.94 \pm 10.62$ & $148.96 \pm 8.03$ & $<0.001$ \\
Weight $(\mathrm{kg})$ & $39.72 \pm 15.53$ & $54.09 \pm 13.57$ & $<0.001$ \\
BMI $\left(\mathrm{kg} / \mathrm{cm}^{2}\right)$ & $21.70 \pm 5.56$ & $24.07 \pm 4.40$ & 0.028 \\
BMl-Z & $1.92 \pm 1.79$ & $2.01 \pm 1.13$ & 0.783 \\
\hline
\end{tabular}

Microbiota profiles with different puberty status OTU classification

Using PLS-DA, the non-pubertal and pubertal groups can be distinguished to a certain extent, suggesting that the two groups differed in the classification of the gut microbiota (Fig. 3).

\section{Alpha- and beta-diversity}

Regarding alpha-diversity, there was no significant difference of the Shannon diversity index, Observed OTUs, Faith's phylogenetic diversity and Pielou's evenness based on OTU distribution between non-pubertal and pubertal groups (all $p>0.05$, Table S4).

Beta-diversity also did not differ significantly between these two aforementioned groups after correction for multiple testing (Table S5). By Distance method BrayCurtis and Statistical method PERMANOVA, PCoA analysis illustrated that the gut microbiota samples from the non-pubertal group could not distinguish from the pubertal group [PERMANOVA] F-value: 0.712; Rsquared: 0.008; $p$-value $<0.735$ (Fig. 4a).

\section{Bacterial taxa differences in subjects with different puberty status}

We used Lefse analysis to identify bacteria where the relative abundance was significantly increased or decreased in each phenotypic category. Non-pubertal subjects had members of the Non-pubertal subjects had members of the order Clostridiales, Pasteurellales, family Clostridiaceae, genus Coprobacillus and Haemophilus that were significantly more prevalent than puberty subjects. Also, the pubertal subjects had members of class Betaproteobacteria, order Burkholderiales that were significantly more prevalent than the non-pubertal subjects (Fig. 5).

Spearman correlation analysis was used to detect an impact of BMI-Z on class Betaproteobacteria, order Clostridiales, Pasteurellales, Burkholderiales, family Clostridiaceae, genus Coprobacillus and Haemophilus. The results showed the relative abundance of order Pasteurellales and genus Haemophilus correlate with BMI-Z ( $r=0.223$ and $0.222, p=0.036$ and 0.036 , respectively), whereas other differential bacterial taxa had no association with BMI-Z $(p>0.05)$.

\section{Microbiota profiles during puberty transition Alpha-, beta-diversity and bacterial taxa differences in non- puberty subgroups}

As for the alpha-diversity between younger children and pre-pubertal groups, there was no statistical difference of the Shannon diversity index, observed OTUs, Faith's phylogenetic diversity and Pielou's evenness based on OTU distribution (all $p>0.05$, Table S4). 
Table 2 Clinical characteristics of the study population divided by puberty stages

\begin{tabular}{|c|c|c|c|c|c|c|}
\hline & Younger children (5-8 years-old) & Pre-puberty & Early puberty & Middle puberty & Late puberty & $P$ value \\
\hline$N(n)$ & 18 & 24 & 18 & 14 & 15 & \\
\hline Age (years) & $6.81 \pm 0.74$ & $9.53 \pm 1.05$ & $10.76 \pm 0.95$ & $10.85 \pm 1.41$ & $11.40 \pm 1.05$ & $<0.001$ \\
\hline Gender (male\%) & $66.7 \%$ & $66.7 \%$ & $77.8 \%$ & $35.7 \%$ & $13.3 \%$ & 0.001 \\
\hline Height (cm) & $124.64 \pm 7.55$ & $139.16 \pm 8.05$ & $148.35 \pm 8.06$ & $149.64 \pm 9.84$ & $149.06 \pm 6.50$ & $<0.001$ \\
\hline Weight (kg) & $30.02 \pm 9.17$ & $47.00 \pm 15.46$ & $56.83 \pm 12.53$ & $55.55 \pm 15.77$ & $49.45 \pm 12.18$ & $<0.001$ \\
\hline BMI $\left(\mathrm{kg} / \mathrm{cm}^{2}\right)$ & $18.98 \pm 4.27$ & $23.74 \pm 5.61$ & $25.53 \pm 4.21$ & $24.28 \pm 4.31$ & $22.12 \pm 4.26$ & 0.001 \\
\hline BMI-Z & $1.47 \pm 1.96$ & $2.26 \pm 1.61$ & $2.26 \pm 1.08$ & $2.19 \pm 1.02$ & $1.55 \pm 1.21$ & 0.256 \\
\hline
\end{tabular}

Beta-diversity also did not differ significantly between these two groups (all p >0.05, Table S5). By Distance method Bray-Curtis and Statistical method PERM ANOVA, PCoA analysis illustrated that the gut microbiota samples from the pre-pubertal group could not separate partly from the younger children group [PERMANOVA] F-value: 0.389; R-squared: 0.0096; $p$ value $<0.982$ (Fig. 4b).

Lefse analysis found no differential bacterial taxa between pre-puberty group and non-puberty group $(p>0.05)$.

\section{Alpha-, beta-diversity and bacterial taxa differences in puberty subgroups}

As for the alpha-diversity among the three subgroups at the different puberty stages, the Shannon diversity index, observed OTUs, Faith's phylogenetic diversity and Pielou's evenness based on OTU distribution, there was no significant differences (all $p>0.05$, Table S4).

Beta-diversity also did not differ significantly among the three subgroups after correction for multiple testing

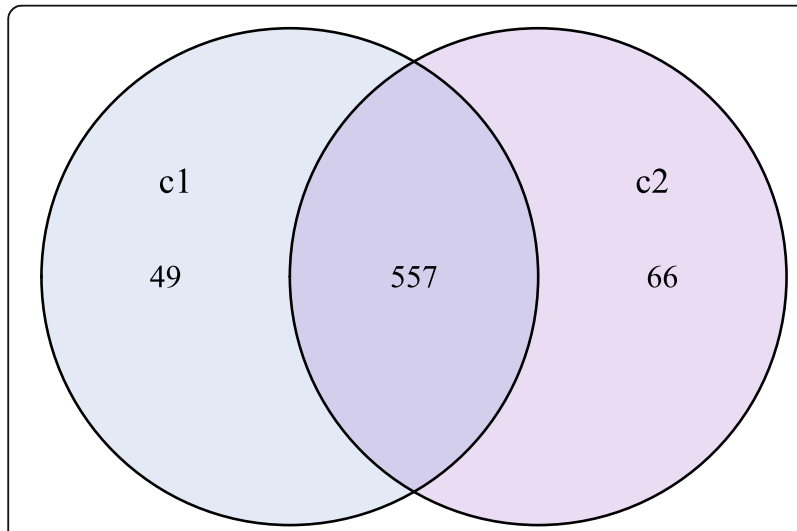

Fig. 1 Shared OTU across the non-puberty and puberty groups. Different colors represent different groups, the interior of each circle represents the number of observed OTUs in the certain group. The overlapping area or intersection represents the set of OTU commonly present in the counterpart groups. Likewise, the singlelayer zone represents the number of OTUs uniquely found in the certain group. C1: puberty group; C2: non-puberty group (all $p>0.05$, Table S5). By Distance method Bray-Curtis and Statistical method PERMANOVA, PCoA analysis illustrated that the gut microbiota samples from the three groups could not distinguish from each other group [PERMANOVA] F-value: 0.857; R-squared: 0.0375; $p$ value $<0.679$ (Fig. 4c).

Lefse analysis found no differential bacterial taxa among early-, middle- and late- puberty group $(p>0.05)$.

\section{Alpha-, beta-diversity in non-puberty and puberty subgroups}

The Shannon diversity index, observed OTUs, Faith's phylogenetic diversity and Pielou's evenness based on OTU distribution did not reveal any significant difference between the pre-puberty and the early puberty groups. Comparing the younger children group and the late puberty group, the Alpha- diversity indexes did not differ, either (all $p>0.05$, Table S4).

Beta-diversity also did not differ significantly between the pre-puberty and the early puberty groups, or the younger children group and the late puberty group. The results were non-significant after correction for multiple testing (all $p>0.05$, Table S5). By Distance method Bray-Curtis and Statistical method PERM ANOVA, PCoA analysis illustrated that the gut microbiota samples from the early puberty group could not distinguish from pre-puberty group [PERMANOVA] Fvalue: 0.70375; R-squared: 0.01729; $p$-value $<0.698$, and the gut microbiota samples from the late puberty group could not distinguish from younger children group, either. [PERMANOVA] F-value: 0.67589; R-squared: 0.021338; p-value < 0.801 (Fig. 4d, e).

\section{Correlations between sex hormone and bacterial abundance}

To evaluate correlations between bacteria and serum sex hormones (testosterone and estradiol), Spearman's rank analysis was adopted. In the pubertal subjects, the abundance of genera Adlercreutzia, Ruminococcus and Dorea was positively associated with the level of testosterone $(r=0.371,0.471$ and $0.488, p=0.040,0.007$ and 0.005 , respectively), and the abundance of genera Parabacteroides 


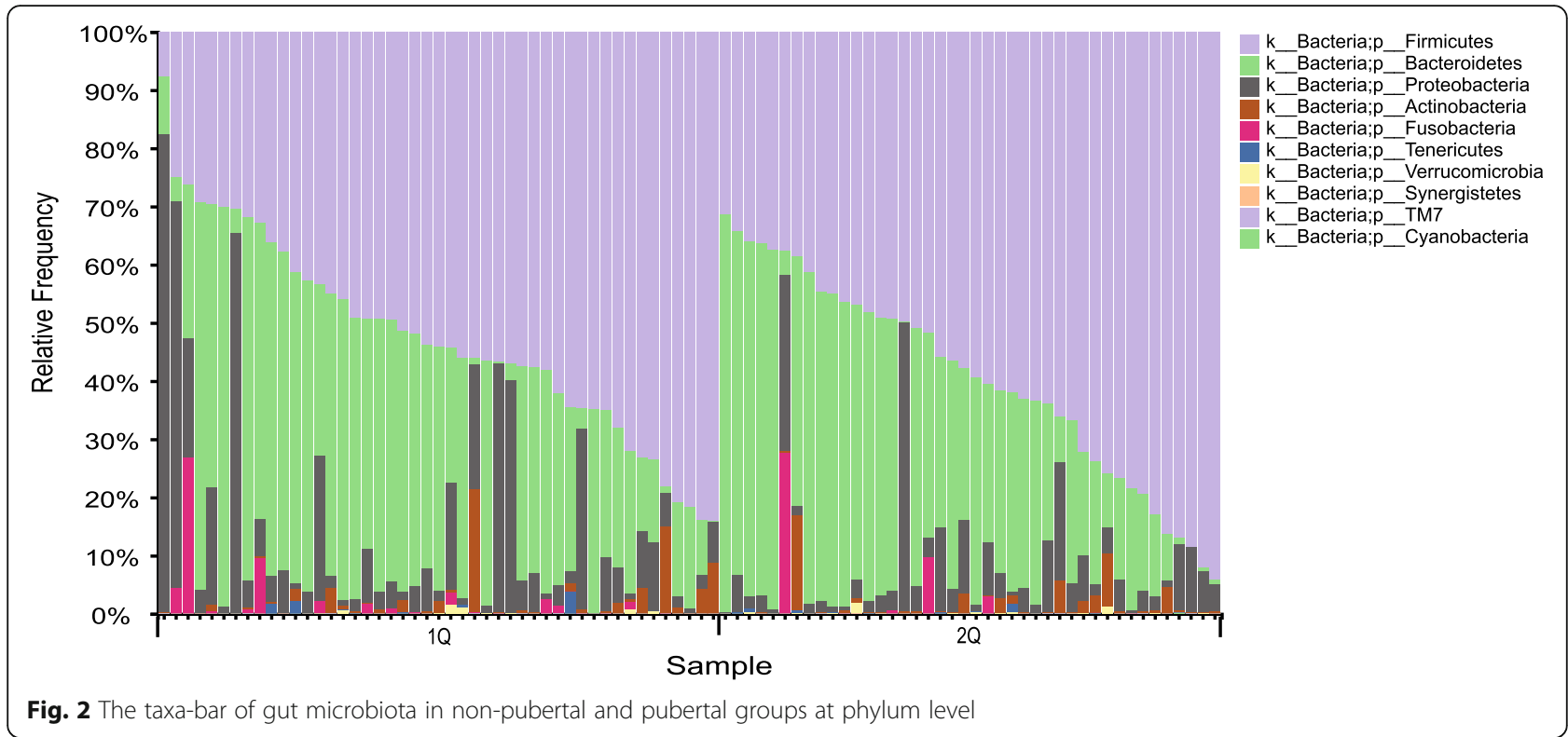

and Clostridium was positively associated with the level of testosterone $(r=-0.424$ and $-0.361, p=0.017$ and 0.046 , respectively). There was no association between the bacterial abundance and serum estradiol (all $p>0.05$ ).

\section{Discussion}

Recent studies have yielded insight into the intricate interactions between age and the gut microbiota by $16 \mathrm{~S}$ rRNA sequencing [10]. With increasing age, it has been observed a gradual and steady reduction in the

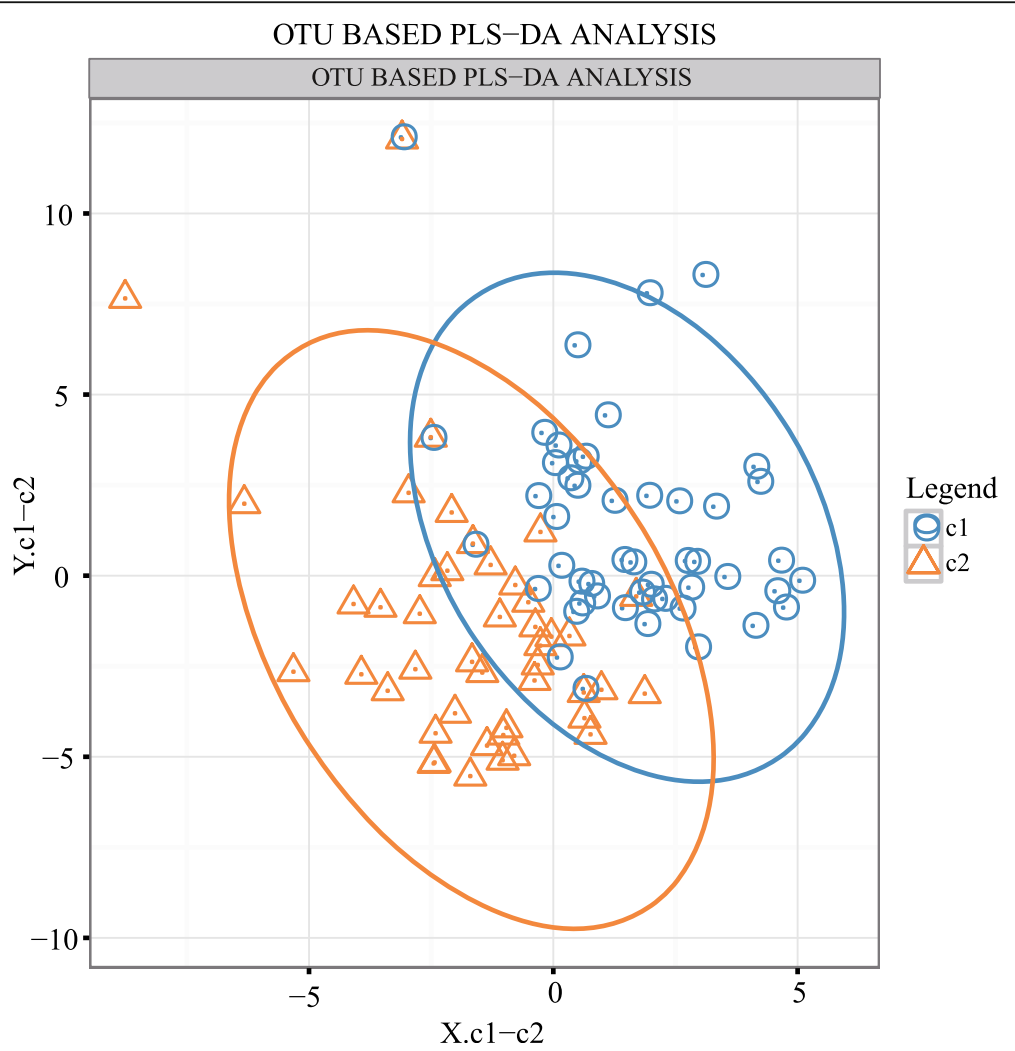

Fig. 3 PLS-DA based on OTU abundance. The horizontal axis and the vertical axis indicate the top 2 components. Each dot denotes one sample. Samples are colored and grouped by elipse according to their group information. C1: puberty group; C2: non-puberty group 


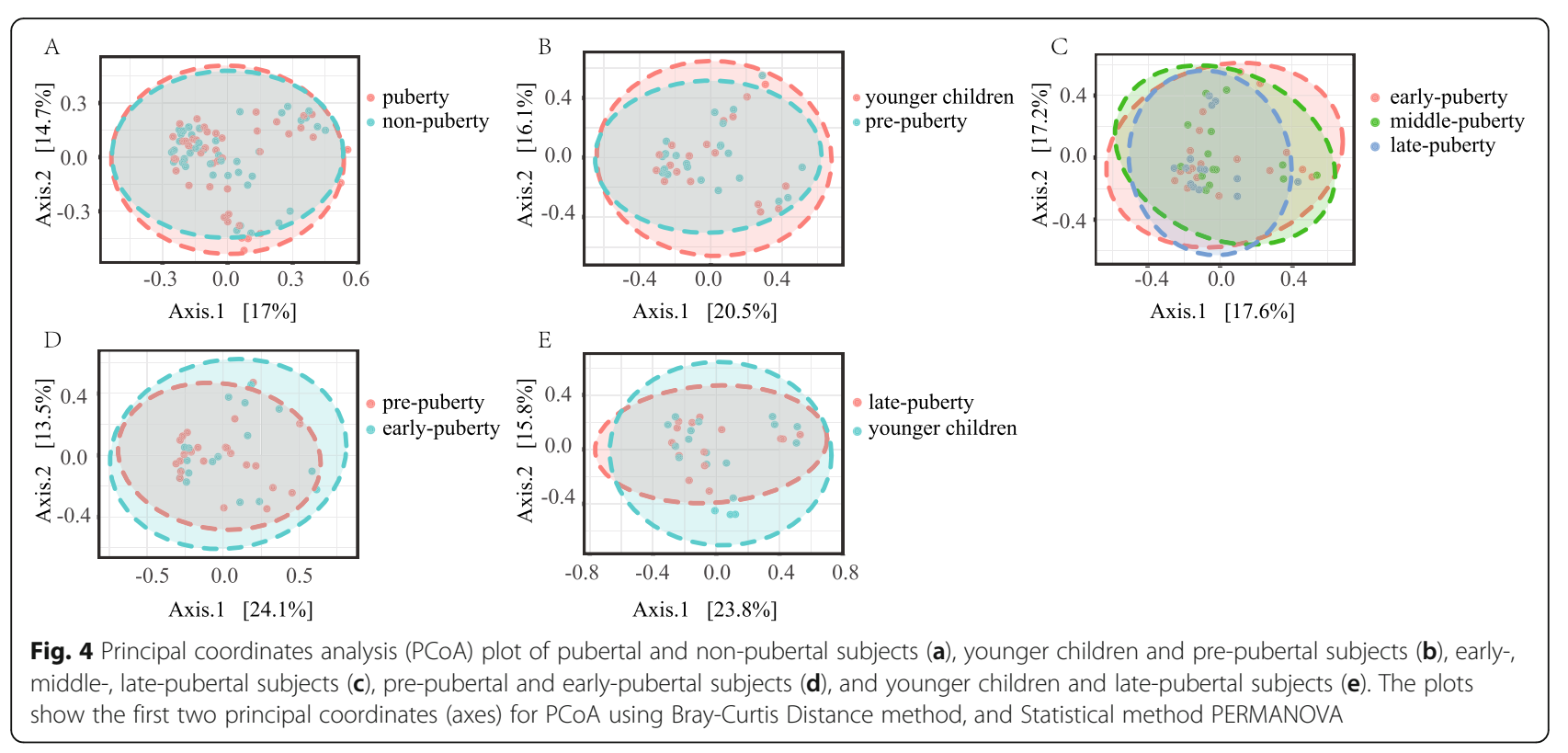

population of aerobes and facultative anaerobes, in concert with a simultaneous upsurge in the number of anaerobes [5, 11]. Previous study reported that the adolescent microbiota was found to differ functionally from that of adults, expressing genes related to development and growth [7], whereas the adult microbiota was more associated with obesity and inflammation [3, 5]. However, the make-up of gut microbiota at different puberty stages were not described [12].

Previous research inferred that during childhood there was less diversity in the gut microbial community compared to adults [13]. In this study, we found no difference in alpha- or beta-diversity between non-pubertal and pubertal subjects. However, differential abundance testing showed differential bacterial taxa between nonpubertal and pubertal subjects. Few studies have addressed differences in gut microbiota as children age [14]. A recent high-throughput analysis of distal gut microbiota found that even though adolescents apparently share a core microbiota configuration with that of adults, they harbor a less complex and considerably different microbiota: Compared with adults, the abundance of Clostridia, Bifidobacterium and Clostridium genera were significantly higher in adolescents $[2,5,6]$. Furthermore, Paliy $\mathrm{O}$ et al. developed a microbiota microarray and found that compared with adults, fecal samples from children harbored more Bacteroidetes and Proteobacteria, and less Clostridia. A number of other putative differences were also reported at the genus level [15].

Specifically, this study found that Non-pubertal subjects had members of the order Clostridiales, family Clostridiaceae, genus Coprobacillus that were significantly more prevalent than puberty subjects. Also, the pubertal subjects had members of order Burkholderiales that were significantly more prevalent than the nonpubertal subjects, and the relative abundance of them were independent of BMI-Z. It has been reported that the abundance of gut Coprobacillus was enriched in $\mathrm{BALB} / \mathrm{c}$ male mice [16], suggesting a possibility that the abundance of gut Coprobacillus could be affected by sex hormone. Dong $\mathrm{G}$ reported that gut Coprococcus was enriched in girls with idiopathic central precocious puberty [17]. All microbes in genera Coprococcus promote SCFAs production [18-20]. Therefore, it is plausible that SCFAs-producing bacteria are increased in pubertal subjects to promote the expression of the leptin gene, which in turn activate the hypothalamic-pituitary-gonad axis, and lead to the onset of puberty [16]. However, we didn't find the abundance of gut Coprobacillus changed between the pubertal and non-pubertal groups.

In addition to the bursts of gonadotropin-releasing hormone with puberty, many other circulating hormones come of age. These profound hormonal changes may be accompanied by changes in the composition of gut microbiota. In a study from the Netherlands, 61 children aged 2-18 years collected fecal samples weekly for 6 weeks, along with a follow-up sample after 18 months. The microbial composition stability varied per phylum at both short-term and long-term intervals. However, the age span of this study precluded the characterization of the gut microbiota at distinct puberty stages. We found there was no significant difference in alpha-, betadiversity, or the differential bacterial taxa between the younger children and pre-pubertal groups. A study of Asian school-age children took the opinion that the presence and ratios of Bifidobacterium / Bacteroides and 


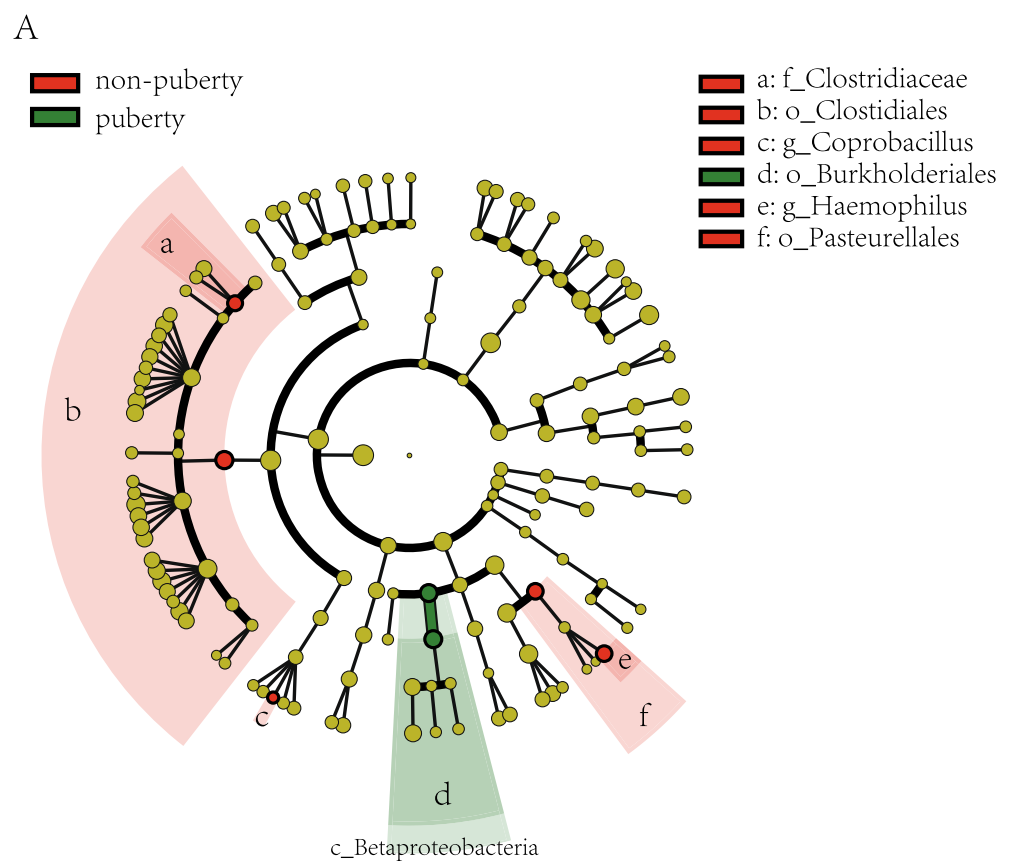

B

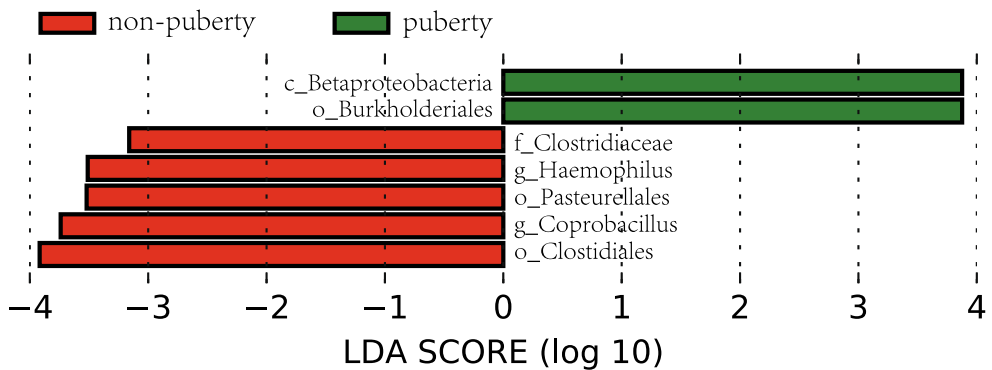

Fig. 5 Differential biomarkers associated with genders in pubertal subjects and non-pubertal subjects. A linear discriminant effect size (LeFse) analysis have been performed (a value $=0.05$, logarithmic LDA score threshold $=2.0$ )

Prevotella enterotype-like clusters were closely associated with geographic regions, because of the varying diets and living environments [21].

Despite the limitations imposed by heterogeneity of the groups, the present study found a relationship between puberty and gut microbiota. During sexual development and growth through the adolescent period, the gut microbiota undergoes progressive changes, likely due to the hormonal surge or other age-related factors. Accordingly, the association between serum sex hormones and bacterial abundance was further analyzed. In the pubertal subjects, it was found that the abundance of genus Adlercreutzia, Dorea, Clostridium and Parabacteroides associated with the level of testosterone. A mouse model study showed that after inoculating germ-free C57BL/6 J mice with fecal bacteria from a man with short-term vegetarian and inulin-supplemented diet, the abundance of Dorea and Clostridium were over- represented in females [22]. Furthermore, Shin JH [23] reported that the abundance of Dorea correlated significantly with the level of testosterone in males, a finding consistent with the results of our study. As known that the two bacteria Parabacteroides and Adlercreutzia could metabolize phytoestrogens with generation of secondary molecules such as secoisolariciresinol, enterolactone, and equol [24], we speculate that the two bacteria might be affected by sex hormones. The association between these bacteria and androgen warrants further investigated.

As a counter-narrative, could the adaptive intestinal microbiome effect serum hormone concentrations or tissue responsiveness [25]? Little is known about the causal inter-relationships between gut microbiota and pubertal development. As differences in gut microbiota become more pronounced at puberty, sex hormones might play an important role in shaping the 
gut microbiota composition is terra incognita [26, 27]. Relevant to our study, transferring the gut microbiota from adult male mice to immature females could result in the levels of testosterone elevated comparable to males, and the recipient's microbiota altered [1]. Interesting to ponder, does a similar phenomenon due to the microbiota exists in humans? It is plausible that microbiota-driven hormone effects are in play and potentially influenced by genetic, metabolic, or psychosocial factors.

This study revealed the diversity of gut microbiota at different puberty stages. However, considering the small sample size and gender differences, for example, between the number of male patients in the prepubertal group and more female patients in the post-pubertal group, render this study at risk of Type 2 error. Even though we found no significant difference of alpha- and beta-diversity between pubertal and non-pubertal groups, a longitudinal study with large sample size wherein the participants are followed over an extended period (pre-puberty to puberty) would confirm a dynamic change in gut microbiome before and after puberty.

\section{Conclusion}

This study is the first report of the characteristics of fecal microbiota during the transitional stages of puberty. Non-pubertal subjects had members of the order Clostridiales, family Clostridiaceae, genus Coprobacillus that were significantly more prevalent than puberty subjects. Also, the pubertal subjects had members of class Betaproteobacteria, order Burkholderiales that were significantly more prevalent than the non-pubertal subjects. Their relative abundance was independent of BMI-Z In the pubertal subjects, it was found that the abundance of genera Adlercreutzia, Dorea, Ruminococcus, Clostridium and Parabacteroides was associated with the level of testosterone. The explanation for the differences in these gut microbiota, and their potential metabolic and hormonal impact, requires additional study.

\section{Methods}

\section{Study population}

This study consisted of 89 children, aged between 5 to 15 years, managed by Fuzhou Children's Hospital of Fujian Medical University as previously described [28].

\section{Dietary assessment}

A semi-quantitative food frequency questionnaire, developed according to the dietary habits of South China, were completed by all participants. Dietary habits during the preceding month were assessed as previously described [28].

\section{Clinical assessment}

Weight and height collected with duplicate measurements on standardized equipment were used to calculate BMI. BMI-Z scores were calculated based on Li Hui et al's reference values, and the diagnostic criteria for obesity or normal weight of Chinese children were as published [29]. Puberty stage was defined according to the Tanner scale by the professionally trained pediatric endocrinologists. Subjects were divided into nonpubertal and pubertal groups. The former group was further subdivided into younger children (5-8 years old) and pre-puberty (Tanner stage $1,>8$ years old) group, and the puberty group was sub-divided into early (Tanner 2), middle (Tanner 3) and late (Tanner 4 and 5) stages for multi-point analysis. All participants maintained their usual dietary habits at least 3 days before blood sampling, and blood samples were collected from each participant after $12 \mathrm{~h}$ of fasting. Blood samples were stored at $-80^{\circ} \mathrm{C}$ and analyzed within 2 weeks. Serum levels of estradiol (E2) and testosterone (T) were measured by chemiluminescent immunoassays (IMMULITE 2000, Siemens Healthcare Diagnostics Products Limited, Germany) using specific reagents.

\section{Fecal sample collection and processing}

The parents of all participants were asked to collect the stool samples in standard collection tubes, and then transported immediately (within $2 \mathrm{~h}$ ) at room temperature for storage at $-80^{\circ} \mathrm{C}$ until further analysis.

\section{Genomic DNA extraction and high throughput sequencing}

MagPure Stool DNA KF kit B (Magen, China) were used for extracting the microbial community DNA following the manufacturer's instructions, and Qubit $^{\oplus}$ dsDNA BR Assay kit (Invitrogen, USA) with a Qubit Fluorometer was used for DNA quantified and the quality was checked by $1 \%$ agarose gel.

Variable regions V3-V4 of bacterial $16 \mathrm{~s}$ rRNA rDNA libraries were prepared by polymerase chain reaction and high-throughput sequencing was performed as previously described [28].

\section{Statistical analysis}

The clinical characteristics of all individuals including anthropometric parameters, levels of E2 and $\mathrm{T}$, and other characteristics were statistically calculated using independent samples $\mathrm{t}$ test, Mann-Whitney $\mathrm{U}$ test, Kruskal-Wallis test and chi-square test by the Statistical Package for the Social Sciences software version 23.0 (SPSS Inc. Chicago, IL, USA). The normality of the data 
was tested using the Kolmogorov-Smirnov test. Clinical data are expressed in means \pm standard error. $P<0.05$ was considered statistically significant.

Statistical analyses of $16 \mathrm{~s}$ rRNA sequencing data were performed on alpha- and beta- diversity measurements by software QIIME2 (v2019.7) [30] as previously described [28]. Kruskal-Wallis Test was used for two groups comparison. Based on the OTU abundance, OTU of each group was listed. Venn diagram was drawn by Venn Diagram of software R (v3.1.1), and the common and specific OTU ID were summarized. Partial least squares discrimination analysis (PLS-DA) completed by package 'mixOmics' of software R. Linear discriminant analysis effect size (LEfSe, v1.0) was used to elucidate significantly different relative abundances of bacterial taxa, associated with different pubertal status. These analyses are presented in a bar plot and the parameters set with an LDA score of 2.0 with LEfSe [31].

\section{Supplementary Information}

Supplementary information accompanies this paper at https://doi.org/10. 1186/s12866-020-02021-0.

Additional file 1: Table S1. Dietary habits of the study population divided by puberty status (Chi-square test). Table S2. Sex hormone concentration of the study population as per puberty stage. Table S3. The absolute abundance of gut microbiota in non-puberty and puberty groups at phylum level. Table S4. Comparison of alpha-diversity between different puberty staging groups. Table S5. Comparison of betadiversity between different puberty staging groups.

\section{Abbreviations}

LEfSe: Linear discriminant analysis effect size; PLS-DA: Partial least squares discrimination analysis

\section{Acknowledgements}

The authors are grateful to all the participants.

\section{Authors' contributions}

$X Y$ drafted the initial manuscript; RMC conceptualized and designed the study, and reviewed and revised the manuscript; $Y Z$ and $X H Y$ collected cases; XQL did the laboratory testing.

\section{Funding}

This study was supported by Technology Innovation Team Train Project of Fuzhou Health Committee in China (2016-S-wp1), and sponsored by key Clinical Specialty Discipline Construction Program of Fuzhou, Fujian, P.R.C (201610191) and Fuzhou Children's Medical Center (2018080310).

\section{Availability of data and materials}

The original contributions presented in the study are publicly available. The raw sequence data reported in this paper have been deposited in the Genome Sequence Archive (Genomics, Proteomics \& Bioinformatics 2017) in National Genomics Data Center (Nucleic Acids Res 2020), Beijing Institute of Genomics (China National Center for Bioinformation), Chinese Academy of Sciences, under accession number CRA003010 that are publicly accessible at https://bigd.big.ac.cn/gsa.

\section{Ethics approval and consent to participate}

This study was reviewed and approved by the Ethics Committee of Fuzhou Children's Hospital of Fujian Medical University, and was conducted in agreement with the Declaration of Helsinki Principles. Written informed consent was obtained from a parent or guardian on behalf of any participants under the age of 16 .
Consent for publication

Not applicable.

\section{Competing interests}

The authors declare that they have no competing interests.

Received: 3 August 2020 Accepted: 26 October 2020

Published online: 03 November 2020

\section{References}

1. Kundu P, Blacher E, Elinav E, Pettersson S. Our gut microbiome: the evolving inner self. Cell. 2017;171(7):1481-93.

2. Yatsunenko T, Rey FE, Manary MJ, Trehan I, Dominguez-Bello MG, Contreras $M$, et al. Human gut microbiome viewed across age and geography. Nature. 2012;486(7402):222-7.

3. Stewart CJ, Ajami NJ, O'Brien JL, Hutchinson DS, Smith DP, Wong MC, et al. Temporal development of the gut microbiome in early childhood from the TEDDY study. Nature. 2018;562(7728):583-8.

4. Hollister EB, Riehle K, Luna RA, Weidler EM, Rubio-Gonzales M, Mistretta TA, et al. Structure and function of the healthy pre-adolescent pediatric gut microbiome. Microbiome. 2015;3:36.

5. Enck P, Zimmermann K, Rusch K, Schwiertz A, Klosterhalfen S, Frick JS. The effects of maturation on the colonic microflora in infancy and childhood. Gastroenterol Res Pract. 2009;2009:752401.

6. Agans R, Rigsbee L, Kenche H, Michail S, Khamis HJ, Paliy O. Distal gut microbiota of adolescent children is different from that of adults. FEMS Microbiol Ecol. 2011;77(2):404-12.

7. Yahfoufi N, Matar C, Ismail N. Adolescence and aging: impact of adolescence inflammatory stress and microbiota alterations on brain development, aging, and Neurodegeneration. J Gerontol A Biol Sci Med Sci. 2020;75(7):1251-7.

8. Robert GH, Luo Q, Yu T, Chu C, Ing A, Jia T, et al. Association of Gray Matter and Personality Development with increased drunkenness frequency during adolescence. JAMA Psychiatry. 2020;77(4):409-19.

9. Integrative HMP. (iHMP) research network consortium. The Integrative human microbiome project. Nature. 2019;569(7758):641-8.

10. Xu C, Zhu H, Qiu P. Aging progression of human gut microbiota. BMC Microbiol. 2019;19(1):236

11. Strati F, Di Paola M, Stefanini I, Albanese D, Rizzetto L, Lionetti P, et al. Age and gender affect the composition of fungal population of the human gastrointestinal tract. Front Microbiol. 2016;7:1227.

12. de Meij TG, Budding AE, de Groot EF, Jansen FM, Frank Kneepkens CM, Benninga MA, et al. Composition and stability of intestinal microbiota of healthy children within a Dutch population. FASEB J. 2016;30(4):1512-22.

13. Ringel-Kulka T, Cheng J, Ringel Y, Salojärvi J, Carroll I, Palva A, et al. Intestinal microbiota in healthy U.S. young children and adults--a high throughput microarray analysis. PLoS One. 2013;8(5):e64315.

14. Flannery J, Callaghan B, Sharpton T, Fisher P, Pfeifer J. Is adolescence the missing developmental link in microbiome-gut-brain axis communication? Dev Psychobiol. 2019;61(5):783-95.

15. Paliy $\mathrm{O}$, Kenche $\mathrm{H}$, Abernathy $\mathrm{F}$, Michail S. High-throughput quantitative analysis of the human intestinal microbiota with a phylogenetic microarray. Appl Environ Microbiol. 2009;75(11):3572-9.

16. Elderman M, Hugenholtz F, Belzer C, Boekschoten M, van Beek A, de Haan $B$, et al. Sex and strain dependent differences in mucosal immunology and microbiota composition in mice. Biol Sex Differ. 2018;9(1):26.

17. Dong G, Zhang J, Yang Z, Feng X, Li J, Li D, et al. The Association of gut Microbiota with Idiopathic Central Precocious Puberty in girls. Front Endocrinol (Lausanne). 2020;10:941.

18. Duncan SH, Belenguer A, Holtrop G, Johnstone AM, Flint HJ, Lobley GE. Reduced dietary intake of carbohydrates by obese subjects results in decreased concentrations of butyrate and butyrate-producing bacteria in feces. Appl Environ Microbiol. 2007;73(4):1073-8.

19. Pryde SE, Duncan SH, Hold GL, Stewart CS, Flint HJ. The microbiology of butyrate formation in the human colon. FEMS Microbiol Lett. 2002;217(2): 133-9.

20. Lin H, An Y, Hao F, Wang Y, Tang H. Correlations of fecal Metabonomic and Microbiomic changes induced by high-fat diet in the pre-obesity state. Sci Rep. 2016;6:21618. 
21. Nakayama J, Watanabe K, Jiang J, Matsuda K, Chao SH, Haryono P, et al. Diversity in gut bacterial community of school-age children in Asia. Sci Rep. 2015;5:8397.

22. Wang JJ, Wang J, Pang XY, Zhao LP, Tian L, Wang XP. Sex differences in colonization of gut microbiota from a man with short-term vegetarian and inulin-supplemented diet in germ-free mice. Sci Rep. 2016;6:36137.

23. Shin JH, Park YH, Sim M, Kim SA, Joung H, Shin DM. Serum level of sex steroid hormone is associated with diversity and profiles of human gut microbiome. Res Microbiol. 2019;170(4-5):192-201.

24. Cady N, Peterson SR, Freedman SN, Mangalam AK. Beyond metabolism: the complex interplay between dietary phytoestrogens, gut bacteria, and cells of nervous and immune systems. Front Neurol. 2020;11:150.

25. Hopkins MJ, Sharp R, Macfarlane GT. Variation in human intestinal microbiota with age. Dig Liver Dis. 2002;34(Suppl 2):S12-8.

26. Yurkovetskiy L, Burrows M, Khan AA, Graham L, Volchkov P, Becker L, et al. Gender bias in autoimmunity is influenced by microbiota. Immunity. 2013; 39(2):400-12.

27. Markle JG, Frank DN, Mortin-Toth S, Robertson CE, Feazel LM, RolleKampczyk $U$, et al. Sex differences in the gut microbiome drive hormonedependent regulation of autoimmunity. Science. 2013;339(6123):1084-8.

28. Yuan $X$, Chen $R$, Zhang $Y$, Lin $X$, Yang $X$. Sexual dimorphism of gut microbiota at different pubertal status. Microb Cell Factories. 2020;19(1):152.

29. Li H, Zong XN, Ji CY, Mi J. Body mass index cut-offs for overweight and obesity in Chinese children and adolescents aged 2-18 years. Zhonghua Liu Xing Bing Xue Za Zhi. 2010;31(6):616-20.

30. Bolyen E, Rideout JR, Dillon MR, Bokulich NA, Abnet CC, Al-Ghalith GA, et al. Reproducible, interactive, scalable and extensible microbiome data science using QIIME 2. Nat Biotechnol. 2019 Aug;37(8):852-7.

31. Segata N, Izard J, Waldron L, Gevers D, Miropolsky L, Garrett WS, et al. Metagenomic biomarker discovery and explanation. Genome Biol. 2011; 12(6):R60.

\section{Publisher's Note}

Springer Nature remains neutral with regard to jurisdictional claims in published maps and institutional affiliations.

Ready to submit your research? Choose BMC and benefit from:

- fast, convenient online submission

- thorough peer review by experienced researchers in your field

- rapid publication on acceptance

- support for research data, including large and complex data types

- gold Open Access which fosters wider collaboration and increased citations

- maximum visibility for your research: over $100 \mathrm{M}$ website views per year

At $\mathrm{BMC}$, research is always in progress.

Learn more biomedcentral.com/submissions 